\title{
Free radicals in the pathogenesis of kwashiorkor
}

By M. H. N. GoldeN* and D. Ramdath, Wellcome Trace Element Research Group, Tropical Metabolism Research Unit, University of the West Indies, Kingston 7, famaica

The skeletal spectre of the marasmic child clearly results from a lack of intake of the bulk nutrients. It can be easily and consistently reproduced in any convenient species of experimental animal.

In stark contrast, the other major form of malnutrition, kwashiorkor, has only once been convincingly reproduced in experimental animals. Coward \& Whitehead (1972) produced oedema, fatty liver, skin lesions, hair discoloration and friability, hypoproteinaemia and mental changes in baboons by feeding the animals exactly the same diets that children with kwashiorkor received in Uganda. The authors demonstrated that the syndrome could be reproduced experimentally but made no attempt to discover the factor or factors in the diet responsible for the disease. In other studies the individual features of kwashiorkor had been singly and inconsistently reproduced in animals using a whole variety of, often extreme, experimental manipulations. The conditions necessary to give a 'model' for kwashiorkor oedema, for instance, are quite different from those used to give experimental fatty liver or hair discoloration.

Why should the kwashiorkor syndrome be so difficult to reproduce experimentally? Why have the applied programmes designed to prevent kwashiorkor been so uniformly unsuccessful? The obvious first answer to be considered is that all the hypotheses that have been proposed, tested experimentally and used as the conceptual framework for the applied programmes, are incorrect. Certainly the extant hypotheses of protein deficiency (Williams, 1935), niacin deficiency (Gillman \& Gillman, 1951), antidiurectic-hormone-like action of free ferritin (Srikantia, 1958), dysadaptation to protein deficient stress (Gopalan, 1968), hormonal dysadaptation (Whitehead, 1979), or aflatoxin intoxication (Hendrickse, 1984 ), do not adequately explain more than a few of the features of kwashiorkor listed in Table $\mathbf{I}$. These features must all be reconciled in any unifying hypothesis. Recently we have proposed that kwashiorkor results from an imbalance between the production of free radicals and their safe disposal (Golden, I985). It is the purpose of the present paper to examine the evidence for such a hypothesis.

The hypothesis, illustrated in Fig. I, states that in kwashiorkor, various noxae are imposed on the subject. These noxae produce free-radical-mediated lipid peroxides and toxic carbonyls. Under normal metabolic and nutritional

-On secondment from Department of Medicine, University of Aberdeen, Scotland. 
Table $\mathrm{I}$. Observations made that must be reconciled in any theory of the pathogenesis and aetiology of kwashiorkor

I. The association of oedema, fatty liver, skin lesions, hair discoloration and mental changes, suggesting a common mechanism.

2. The association with certain staple foods: cassava, yam, plantain, rice, maize, cruciferae.

3. The lack of kwashiorkor in 'primitive' cultures where the staple foods, associated in other cultures with kwashiorkor, are prepared in the traditional manner such as fermentation (cassava, breadfruit), or by the addition of burnt plant ash (maize).

4. The precipitation of kwashiorkor by infection, particularly measles, tuberculosis, malaria and diarrhoea.

5. The almost universal presence of infection and overgrowth of bacteria in the small intestine.

6. The unpredictable fluctuation in prevalence from year to year in relation to crops, harvest and climate.

7. The association with humid climates and wet seasons.

8. Occurrence predominantly in the newly weaned child.

9. A relatively small proportion of children consuming a 'kwashiorkor-associated diet' actually develop the disease $(0.5-2 \%)$.

10. The occasional occurrence of kwashiorkor in the very young child and in the fully breast-fed child.

II. It is extremely difficult to produce under controlled, hygienic conditions with a full diet lacking single essential nutrients (or classes of nutrients).

12. Skin lesions are particularly florid in dark skinned patients in the tropics.

13. Associated with low levels of hepatic export proteins, high levels of circulating ferritin and vitamin $B_{12}$, high levels of hepatic iron whilst all the other trace metals, RNA, and soluble protein in the liver are reduced.

14. Associated with low concentrations of vitamins $\mathrm{E}$ and $\mathrm{A}$, carotene, zinc and selenium.

15. Associated with a consistently low erythrocyte glutathione (reduced) (GSH), high peroxide haemolysis, low NADPH:NADP ratio, low glutathione peroxidase (EC 1.I I.I.9), induced glutathione-S-transferase (EC 2.5.1.18) and hexose-monophosphate shunt.

16. Leaky cell membranes with altered lipid profile, an increased sodium pump and intracellular $\mathrm{Na}$ with decreased potassium.

17. Loss of oedema on a minimal diet containing a lower concentration of protein than the antecedent diet.

I8. Usual response to admission to hospital with treatment of infection, provision of a hygienic environment and a properly prepared milk-based diet.

19. Usual complete recovery leaving no pathological lesions, with subsequent growth and metabolic responses that are not different from peers who never had kwashiorkor.

circumstances, provided that the flux of radicals is not too intense, the radicals are scavenged and dissipated through the appropriate protective pathway. The flux of radicals is increased with abundant storage iron, or at least in the absence of $\mathrm{Fe}$ deficiency; this is because $\mathrm{Fe}$, being a redox catalyst, both multiplies the number of radicals produced and creates more reactive and damaging species, unless it is strictly compartmentalized and bound to a restrictive chelator. 


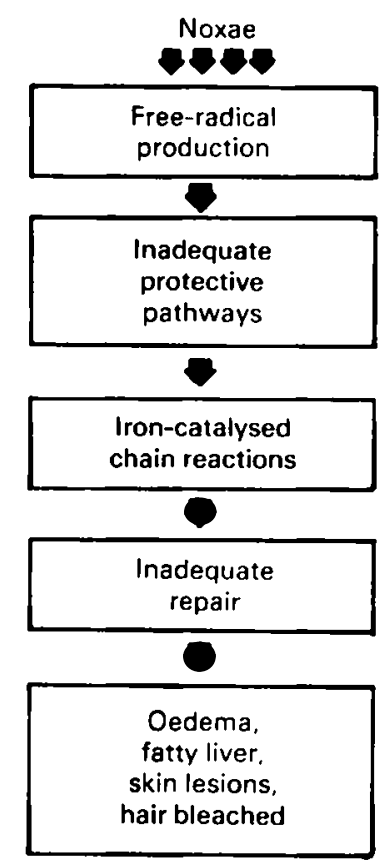

Fig. 1. Proposed steps in the pathogenesis of kwashiorkor.

The reason why the radicals and their products result in kwashiorkor is that there is a relative, and probably also an absolute, general deficiency of the protective mechanisms. Most of the protective pathways require micronutrients: a severe dietary deficiency of any one of these nutrients will lead to loss of free radical protection in some cellular compartment. Even a mild deficiency of several of the micronutrients is likely to lead to a general lack of protection, so that the balance between radical production on the one hand and dissipation, repair of damage and safe disposal of the toxic products of free radical action on the other hand, cannot be redressed. The resulting damage then gives rise to the oedema, fatty liver, pigmentary changes, diarrhoea, immunoincompetence and mental changes, typical of kwashiorkor. The steps of the hypothesis are now discussed.

\section{Free-radical-producing noxae in kwashiorkor}

Infection. Infection is almost ubiquitous in kwashiorkor (Morehead et al. 1974): kwashiorkor is frequently precipitated by an infection such as measles (Morley, 1964). The body's defence against invading organisms is to produce free radicals in sufficient quantities to kill the organisms. The body relies upon its own protective mechanisms to limit the extent of self-damage and to repair the unavoidable damage after the organism is killed. Thus, stimulated leucocytes produce large quantities of superoxide and hydrogen peroxide (Gabig \& Babior, 1982), which they release into the surrounding medium. Peroxy lipids are formed which 
stimulate further leucocytes (McCord \& Petrone, 1982). Infections and inflammatory toxins are thus potent stimulators of free radical formation.

Toxins. Kwashiorkor occurs in areas with inadequate food handling and storage facilities, and in times of food shortage when spoilt foods are likely to be consumed. In these areas most weaning foods are heavily contaminated with toxogenic bacteria (Rowland et al. 1978; Hibbert \& Golden, I981). A whole range of toxogenic fungi grow on stored foods in Third World communities. Attention has been focused upon one of these, aflatoxin, which should probably be regarded as an index of general mycotoxin exposure rather than as a specific intoxication. Aflatoxin concentration has been found to be very high in the blood and liver of children with kwashiorkor (Hendrickse, 1984). Although the exposure to this toxin may not be greater in the child with kwashiorkor than in the child with marasmus, as the urinary excretion of aflatoxin metabolites is equally high in both conditions, clearly, the child with kwashiorkor is not able to safely dispose of the toxin after it is metabolized to an epoxy derivative.

The overgrowth of the children's small intestine with bacteria constitutes an uncontrolled biochemical powerhouse capable of producing numerous products (Drasar \& Hill, 1974), many of them toxic. Small bowel overgrowth (Heyworth \& Brown, 1975) and endotoxaemia (M. H. N. Golden, D. Ramdath and C. Murphy, unpublished results) are usual in kwashiorkor. Fatty liver, a hallmark of hepatic free radical damage, is produced by small bowel overgrowth both clinically and experimentally. Indeed the hepatic lesions, changes in intermediary metabolism and sensorial effects of severe endotoxaemia are very similar to the changes seen in kwashiorkor (Cooperstock et al. 1975).

It is clear that kwashiorkor is associated with an enormously increased stress from noxae that produce free radicals.

\section{Catalysis of free radical production}

The major catalyst of free radical reactions in vivo is $\mathrm{Fe}$ (Halliwell \& Gutteridge, 1984). This is because of its relative abundance and the ease with which it changes its valency state under in vivo conditions of oxidation-reduction potential and $\mathrm{pH}$; Fe acts as a catalyst by redox cycling with reducing equivalents such as ascorbic acid and NADPH. In the presence of $\mathbf{F e}$ a single radical can readily inititate a chain reaction with the formation of many radicals and their peroxy and carbonyl products (Mead et al. I982).

The question therefore arises as to whether Fe stores are high or low in children with kwashiorkor. Fig. 2 shows the hepatic Fe concentrations compared with the concentrations of other trace metals in Jamaican children dying from kwashiorkor. Similarly high hepatic $\mathrm{Fe}$ has been found in children from Lebanon (McLaren et al. 1968), India (Mukherjee \& Sarkar, 1958) and South Africa (Gillman \& Gillman, I95 I). Furthermore, when marrows from children in these countries have been examined, they have been found to contain abundant Fe (Adams \& Scragg, 1965). 


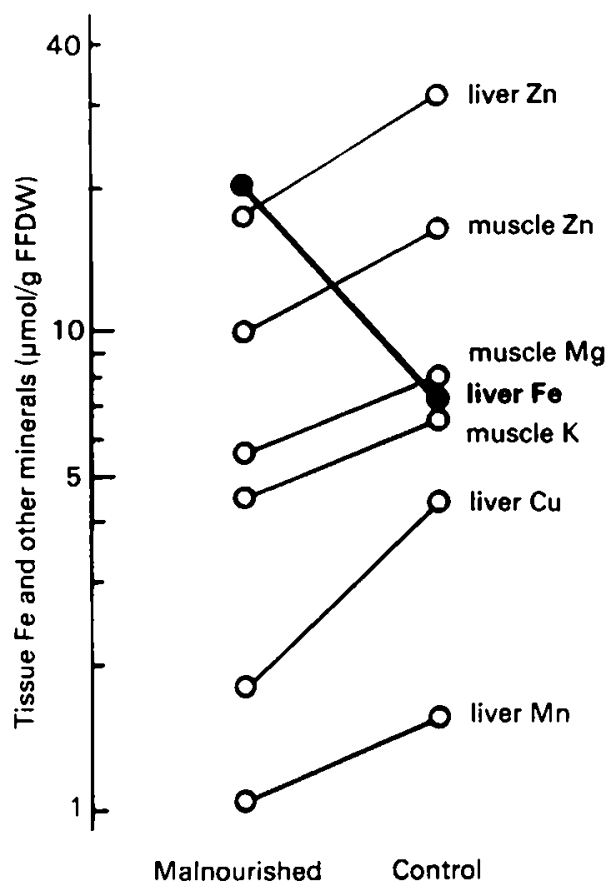

Fig. 2. The concentration of metals in the liver and muscle of malnourished and either control children or children recovered from malnutrition. Liver: zinc, copper, manganese (Warren et al. 1969); iron (Waterlow, 1948). Muscle: $\mathrm{Zn}$, magnesium (Cheek et al. 1970); potassium (Nichols et al. I969). The values have been converted to a fat free dry weight (FFDW) basis where appropriate: $\mathrm{K}_{10} 0^{-2}, \mathrm{Mg}^{\circ} \mathrm{O}^{-1}$.

We have recently measured plasma ferritin in severely malnourished children: it is increased (Golden et al. 1985), particularly in those children who die. It is likely that children with kwashiorkor do not have Fe deficiency, and may indeed usually have an $\mathrm{Fe}$ overload. This $\mathrm{Fe}$ may well be available as a redox catalyst, particularly as the synthesis of transferrin and other iron Fe-binding proteins is impaired in kwashiorkor.

In any chronically malnourished population only about $0.5^{-2} \%$ of the children actually develop kwashiorkor, despite the harsh conditions under which most of the children live. It is tempting to speculate that it is the few individuals in the tail of the population distribution of $\mathrm{Fe}$ status that have high $\mathrm{Fe}$ stores that are thus predisposed to develop kwashiorkor. In this respect one can envisage that $\mathbf{F e}$ deficiency, in a hostile environment, is protective with respect to kwashiorkor, much as sickle cell anaemia or glucose-6-phosphate dehydrogenase (EC 1.1.1.49; G6PD) deficiency is protective against malaria. Of course in a 'Western' environment all these conditions are relatively deleterious. 


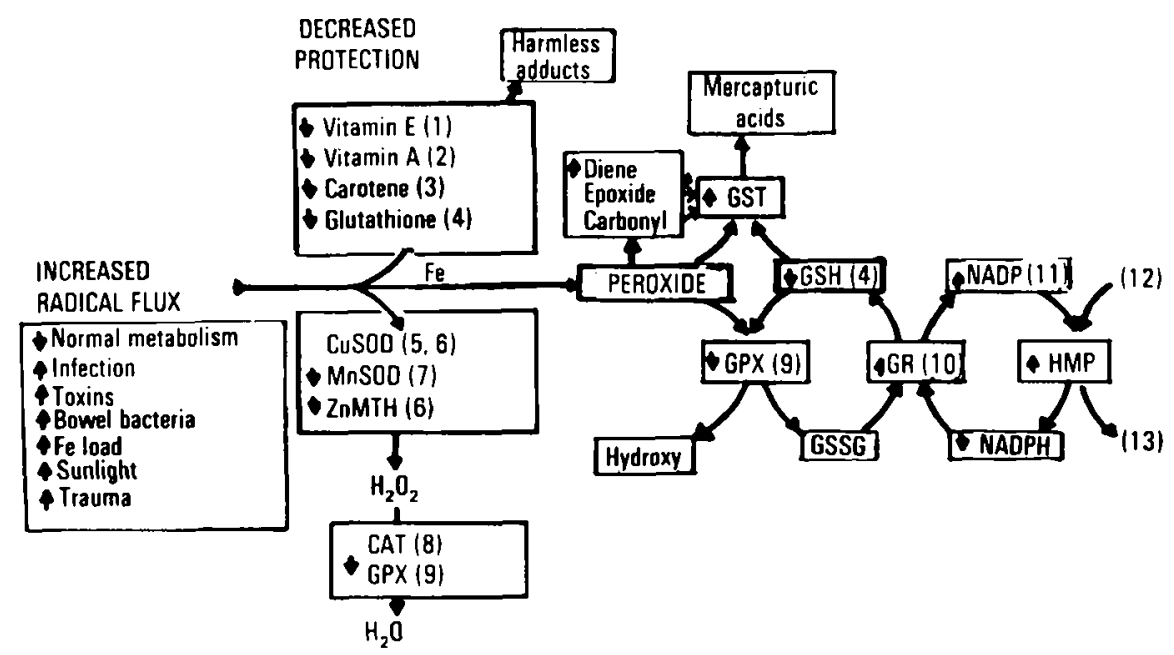

Fig. 3. Diagram showing the mechanisms of radical production and subsequent metabolism. $\uparrow . \downarrow$ beside the substrates, products and enzymes show whether they have been demonstrated to be increased or decreased respectively in children with kwashiorkor. The essential nutrients involved are: (I), vitamin $\mathrm{E} ;(2)$, vitamin $A ;(3)$, carotene; (4), sulphur amino acids; (5), copper; (6), zinc; (7). manganese; (8), iron; (9), selenium; (10), riboflavin; (I I), nicotinic acid; (12), magnesium and phosphorus; (13), thiamin.

CuSOD, Cu-Zn superoxide dismutase (EC 1.15.1.1); MnSOD, Mn superoxide dismutase: ZnMTH, Zn metallothionein; GST, glutathione-S-transferase ( $E C$ 2.5.1.18); GPX, glutathione peroxidase (EC 1.11.1.9); GSH, glutathione (reduced); GSSG. glutathione (oxidized); GR, glutathione reductase (EC I.6.4.2): HMP, hexose-monophosphate shunt (glucose-6-phosphate dehydrogenase ( $E C$ 1.1.1.49; G6PD), 6-phosphogluconic acid dehydrogenase (EC 1.1.1.43)); CAT, catalase ( $E C$ I.1 1.1.6).

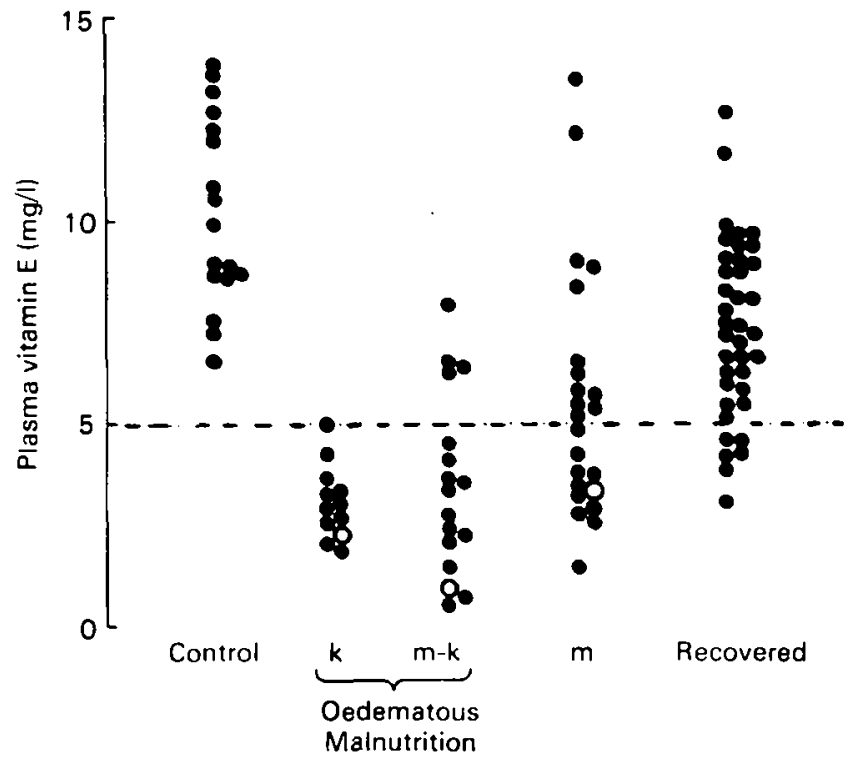

Fig. 4. Plasma vitamin E concentrations in kwashiorkor (k), marasmus (m), marasmus-kwashiorkor $(\mathrm{m}-\mathrm{k})$, recovered and control children, taken within $24 \mathrm{~h}$ of admission. Each point represents a separate child. (- - - ), the commonly accepted limit of normality. (O), Children who subsequently died. 


\section{Protective mechanisms against free radicals in kwashiorkor}

Free radical reactions are one of the basic biochemical mechanisms of the body: free radicals are normal intermediates in metabolism. Thus all mono-oxygenases, and several dehydrogenases, especially xanthine oxidase ( $E C$ I.I.3.22), cytochrome $P_{45} \circ$ and $b_{6}$, prostaglandin synthetase, leucotriene synthetase and the mitochondrial respiratory chain, normally generate superoxide, hydrogen peroxide and free radicals. Radical production is purposely increased as part of any inflammatory reaction by the activity of specific NADPH-dependent lipo-oxygenases. They and their toxic products do not normally cause damage because a whole range of defence mechanisms exists to protect the different parts of the cell. The major protective pathways, the nutrients involved and the changes that occur in kwashiorkor, are summarized in Fig. 3.

Dismuting pathways. In malnutrition, plasma vitamin $\mathrm{E}$, vitamin $\mathrm{A}$ and carotene are each severely reduced, both absolutely and relative to lipid concentrations. This has been consistently shown in all countries where investigations have been made. Vitamins $\mathrm{E}$ and $\mathrm{A}$ were shown by McLaren $e$ al . (1969) to be specifically related to the prognosis and to be useful in the classification of malnourished children. In Jamaica we find that vitamins $\mathrm{E}$ and $\mathrm{A}$ are reduced in both oedematous and non-oedematous malnutrition. Values for vitamin $E$ are shown in Fig. 4; those for vitamin $A$ and carotene show a similar pattern.

These nutrients are bleached and consumed when they dismute a free radical. It is not clear whether the levels are low in kwashiorkor because of an increased rate of consumption by dissipation of radicals or whether they are low because of an inadequate dietary intake. Both mechanisms are probably important. Increased destruction would account for both the very low levels of carotene and vitamin A found in populations that consume yellow maize (e.g. Haiti) and yet develop kwashiorkor with hypocarotenaemia, and also for the devastating progression of symptomatic vitamin A deficiency in children under treatment for kwashiorkor, despite administration of vitamin A (Pereira \& Begum, 1971). It is noteworthy that children who present with kwashiorkor have a lower carotene level than those who present, from the same population, with marasmus (e.g. McLaren et al. 1969) despite consuming similar diets (Gopalan, 1968), because carotenes are particularly susceptible to bleaching by organic free radicals (Bors et al. 1982).

We were only able to demonstrate a reduction in erythrocyte cell copper superoxide dismutase (EC I.15.1.I) in $25 \%$ of malnourished children in Jamaica (Bennett et al. 1984). However, the hepatic levels of $\mathrm{Cu}$ are low in kwashiorkor (Warren et al. 1969), so that the erythrocyte may not be representative of the more vulnerable cells in the body, or there may be regional differences (Golden, 1985).

The mitochondrial enzyme, manganese superoxide dismutase, has not yet been measured in kwashiorkor. However, there is a marked reduction in hepatic manganese in South African children with kwashiorkor (Warren et al. 1969) but not in control or non-oedematous children. This observation is particularly 


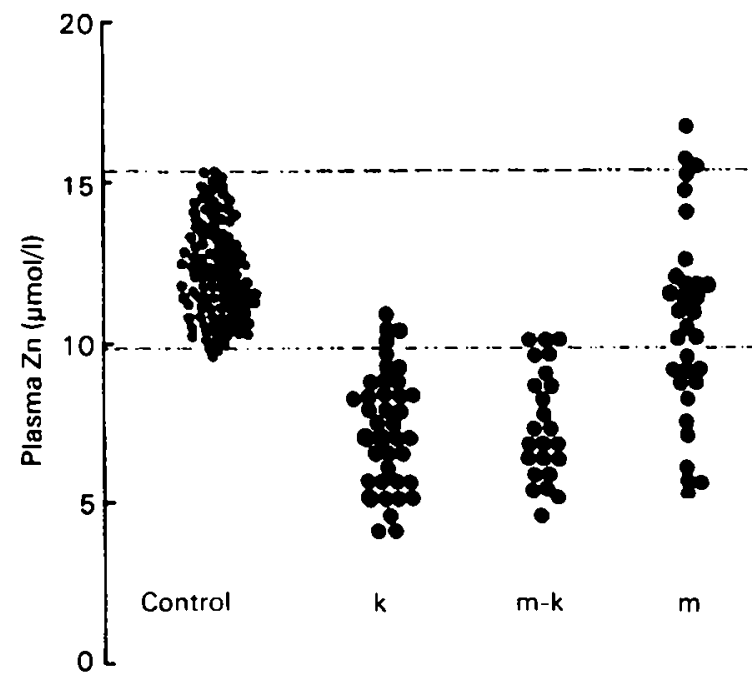

Fig. 5. Admission plasma zinc concentrations in kwashiorkor (k), marasmus (m), marasmuskwashiorkor $(m-k)$ and control children. Each point represents a separate child. (---), the commonly accepted limits of normality.

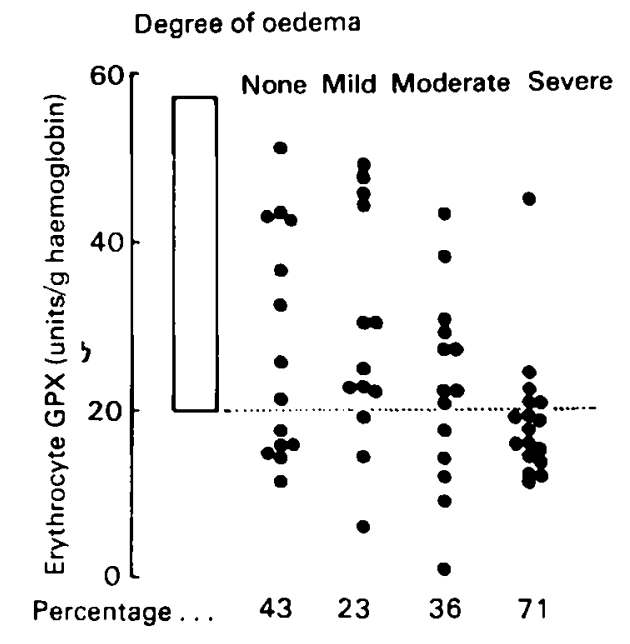

Palpable liver size $(\mathrm{mm})$

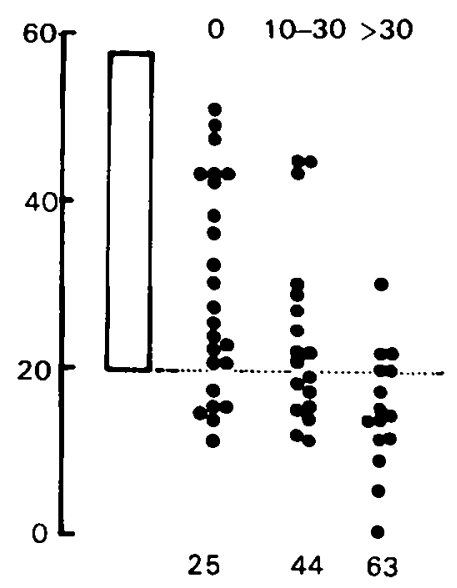

Fig. 6. Admission erythrocyte glutathione peroxidase (EC 1.1 1.1.9; GPX) activity in malnourished children plotted against the degree of oedema and the palpable size of the liver below the mid-clavicular costal margin. No oedema represents marasmus. The block shows the range of normal Jamaican infantile control. The percentage of subjects in each group below the lower limit of the control (- - - ) is shown. 
important as manganese superoxide dismutase is a key enzyme in the protection of mitochondria. The ultrastructural appearance of the liver mitochondria from Mn-deficient experimental animals and from children with kwashiorkor are strikingly similar. Hepatic mitochondrial damage from lack of this enzyme, glutathione peroxidase ( $E C$ I.I I.1.9; GPX) and glutathione itself could account for the accumulation of fat in the liver.

$\mathrm{Zinc}$, in the form of $\mathrm{Zn}$ metallothionein, is a very effective free radical sink in vitro, and probably also in vivo. Although the concentration of intracellular $\mathbf{Z n}$ metallothionein has not yet been measured in malnutrition, circulating zinc levels are uniformly low in kwashiorkor (Fig. 5).

Removal of radical-induced toxic products. The radicals that escape safe dissipation generate peroxides, particularly in cell membranes. The organic peroxides are substrates for the selenium-containing enzyme, GPX, which reduces the peroxide to hydroxyl with oxidation of glutathione. This enzyme is reduced in malnutrition (Fig. 6), particularly in children with more severe disease. Se, itself, has also been shown to be reduced in the blood of children with kwashiorkor from Guatemala (Burk et al. 1967), Thailand (Levine \& Olson, 1970) and Zaire (Fondu et al. 1978). Presumably GPX is reduced in all these populations. The result will be an inefficient removal of organic peroxide. The peroxides will then break down to form toxic aldehydic products such as 4 -hydroxynonenal (Esterbauer, 1982), which may be the most damaging species produced (Slater, 1984). Measurements of these specific carbonyls are not yet available. However, preliminary measurements of plasma concentrations of dienes in a few children with kwashiorkor show that they are about twice those of control and marasmic subjects (D. Ramdath, unpublished results).

The resulting aldehydes, epoxides, other products with electrophilic centres and peroxides themselves are then detoxified by conjugation with one of the family of enzymes collectively termed glutathione-S-transferase (EC 2.5.1.18; GST). The resulting glutathione-S-conjugate is metabolized to a mercapturic acid and excreted either in the bile or the urine. Measurement of erythrocyte GST in forty-one malnourished children showed that this enzyme is induced in kwashiorkor (kwashiorkor 3.23 (SE 0.23); control I.90 (SE 0.12) U/g haemoglobin), with activities above the upper limit of normal in over half the children (Charley et al. 1986).

One major difference between the metabolism of peroxides by GPX and GST is that, with GPX, glutathione functions only as a reducing equivalent: the oxidized glutathione generated can be reduced with glutathione reductase $(E C$ r.6.4.2) to regenerate the reduced glutathione (GSH) so that with GPX there is no net consumption of glutathione. In contrast, with GST there is disposal by formation of a glutathione conjugate and hence there is a net consumption of GSH. Therefore if there is a deficiency of Se and consequently a low GPX activity, peroxides will be predominantly metabolized by GST; this will cause a constant drain on GSH and require greatly increased de novo glutathione synthesis. A sulphur amino acid deficiency could be produced in this way. 


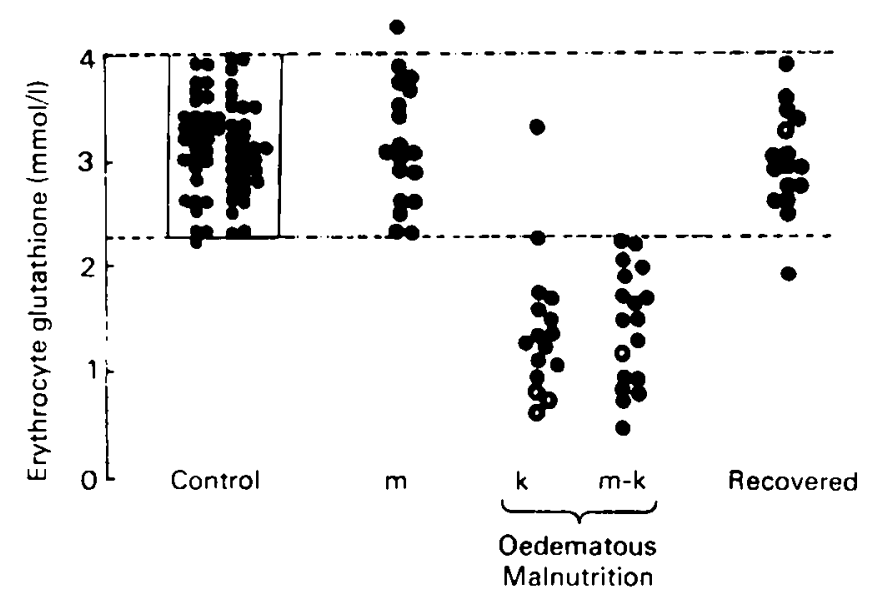

Fig. 7. Admission erythrocyte total glutathione measured by the recycling assay of Tietze (1969). Each point is from a separate child. $m$, Marasmus; $k$, kwashiorkor; $m-k$, marasmus-kwashiorkor. (O), Children who subsequently died. The block shows the range of normal Jamaican infantile control. (- - - ), the commonly accepted limits of normality.

Glutathione. Glutathione is the central component of the whole protective repertoire. Not only is it an effective aqueous free radical scavenger in its own right but it also functions to maintain protein sulphydryl groups in the reduced state and is the cofactor for both of the major detoxification pathways, GST and GPX. Clearly, the GSH status of the malnourished child will be crucial to his ability to withstand a free radical stress, and may indeed determine whether or not kwashiorkor, as opposed to marasmus, will supervene.

Fig. 7 demonstrates that glutathione is specifically reduced in the erythrocytes of children with kwashiorkor, with or without concomitant marasmus. The remarkable thing about these results is that glutathione levels are normal in pure marasmus and low in kwashiorkor with almost no overlap between the diagnoses. The sensitivity and specificity of erythrocyte GSH to differentiate oedematous from non-oedematous malnutrition (ninety-three subjects) are both over $91 \%$. Furthermore, the few 'false negatives' had equivocal or minimal oedema, which disappeared in a day or so; they did not lose any weight on refeeding, they also did not have the skin, hair or liver changes associated with kwashiorkor; they were classified as kwashiorkor, however, because we use the Wellcome classification (Wellcome Trust Working Party, 1970) in which the presence or absence of oedema has primacy in differentiating the conditions. All the children with unequivocal kwashiorkor syndrome had very low levels of glutathione. The oedematous child with normal glutathione had heavy proteinuria and was probably a misdiagnosed nephrotic. All the very sick children had profoundly reduced glutathione concentrations. One can certainly successfully differentiate between kwashiorkor and marasmus on the basis of the erythrocyte glutathione concentration alone.

Longitudinal measurements show that the glutathione levels remain low whilst 
the child is oedematous. When oedema is lost the concentration quickly rises (D. Ramdath and M. H. N. Golden, unpublished results).

The question now arises as to whether the glutathione concentration is low primarily because of increased consumption or whether it is low because of a deficiency of the cysteine, glycine or total nitrogen needed to synthesize glutathione. The diets associated with kwashiorkor are characterized by their low content of sulphur amino acids and protein deficiency is meant to be a major factor in kwashiorkor (sic), so that an amino acid limitation is the obvious first hypothesis. However, this is probably not the correct interpretation.

We took whole blood from sixteen malnourished children and incubated it in vitro at $25^{\circ}$. There was an increase in the whole blood glutathione content of about $20 \%$ over about $4 \mathrm{~h}$ (Fig. 8). This increase was entirely prevented if 5 mM-methionine sulphoximine, a specific inhibitor of glutathione synthase (EC 6.3.2.3), was added to the whole blood. As nothing was added to the incubated whole blood, the observed increase in glutathione implies that there was sufficient cysteine, glycine, glutamine and energy in the whole blood of the malnourished children to synthesize glutathione de novo: hence the substrates for synthesis of glutathione were not limiting in vivo. We conclude that the erythrocyte glutathione was reduced in vivo predominantly because of increased consumption rather than decreased production. The glutathione was almost certainly consumed in detoxifying peroxides and carbonyls that entered the circulation from the tissues. This itself implies that in kwashiorkor there is a greatly increased flux of peroxides requiring disposal. This conclusion is supported by measurements of the other components of the GSH regenerating system (Fig. 3).

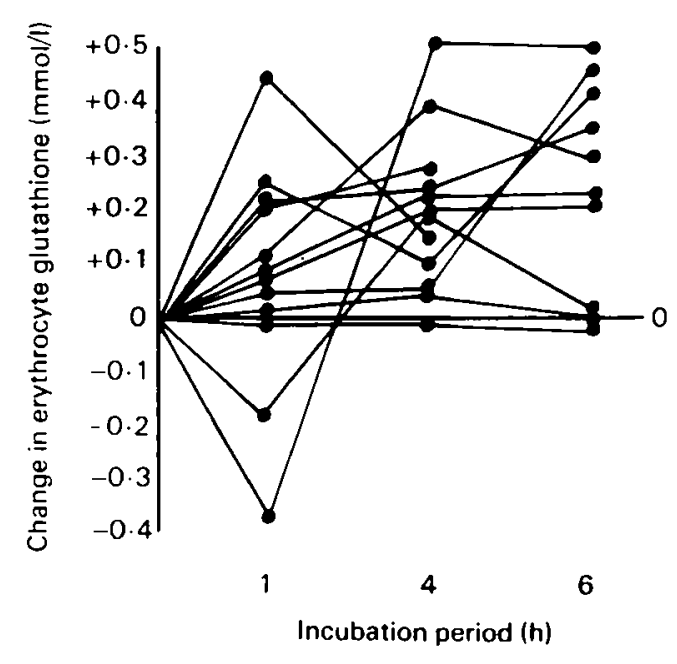

Fig. 8. Change in the whole-blood glutathione concentration, expressed in terms of erythrocytes, in blood taken from twelve malnourished children and incubated at $25^{\circ}$ without any manipulation: at the time-intervals shown ro- $\mu$ l samples were taken in duplicate. Mean (with SE): I h. 0.143 (SE $0.081), 4$ h 0.133 (SE 0.065$), 6$ h $0.258($ SE 0.060). 
The ratio of glutathione (oxidized) (GSSG): GSH is normal in kwashiorkor $(2 \mathrm{GSSG}) /(\mathrm{GSH}+2 \mathrm{GSSG})=3.6 \%$ in both controls and kwashiorkor). However, this ratio is maintained at that level by the very active glutathione reductase, so that this measurement does not serve to give an indication of the rate of oxidation of GSH; it does serve to show that the observed physiological abnormalities are unlikely to be principally due to the formation of mixed disulphides between GSH and protein sulphydryl groups.

The two sequential enzyme systems responsible for glutathione reduction and provision of reducing equivalents respectively are both induced in kwashiorkor (Fig. 3). Thus there are increased activities of glutathione reductase (Fondu et al. I978) and of G6PD and 6-phosphogluconic acid dehydrogenase (M. H. N. Golden, L. Charley and D. Ramdath, unpublished results). With the induction of the hexose-monophosphate shunt one might expect there to be increased levels of NADPH and reduced levels of NADP. In fact the reverse is observed so that despite the increased activity of the hexose-monophosphate shunt, there is a reduced concentration of NADPH and an increased NADP. The total concentration of (NADP + NADPH) is normal (D. Ramdath, unpublished results). This implies that in kwashiorkor there is a greatly increased consumption of NADPH and that the hexose-monophosphate shunt is being driven by the relatively oxidizing redox state. It would appear that, although the activity of GPX is low when measured in vitro, GPX is probably working at near maximum activity in vivo.

Conclusion. It is clear that in kwashiorkor not only is there likely to be an increased flux of free radicals generated by the various noxae, but also virtually all the protective pathways are compromised. Both sides of the balance are upset and the stage is set for free radical damage to occur. Indeed, given the severity and chronicity of the noxae and the widespread and often profound reduction of the protective machinery it is surprising that so many children survive kwashiorkor and return to normality.

That these processes do in fact result in an exposure to increased quantities of peroxides and toxic carbonyls is strongly suggested by the demonstration of excessive plasma dienes, and by the increased consumption rate of erythrocyte glutathione for both conjugate formation and to provide reducing equivalents. Preliminary observations of the electron-spin-resonance spectra of liver biopsies taken immediately after death demonstrate strong, as yet unidentified peaks that originate from some form of free radical (T. F. Slater, M. H. N. Golden and R. Cheeseman, unpublished results); these could be agonal or post-mortem changes (Slater, 1984) and do not, of course, constitute definitive proof of a free radical excess during the genesis of kwashiorkor.

\section{Damage produced by unsuppressed radical peroxidation}

That oedema can result from free-radical-mediated mechanisms is demonstrated by the oedema that occurs in vitamin-E-deficient premature infants stressed by a diet high in polyunsaturated fats (Hassan et al. 1966): indeed, this oedema is very 
similar in behaviour and distribution to the oedema of kwashiorkor. However, if free-radical-mediated damage is the cause of oedema, the precise mechanism is not clear.

Peroxidation of membranes results in their becoming relatively leaky. This leakiness has been observed in leucocytes (Patrick \& Golden, 1977) and in erythrocytes (Kaplay, 1978) in kwashiorkor but not in marasmus; there is an increased number of sodium pumps and an increased rate of $\mathrm{Na}$ pumping, despite a reduced intracellular potassium and a raised intracellular $\mathrm{Na}$. If similar membrane defects occur in the capillary and renal cell membranes as are found in the blood cells, this may account for the increased renal reabsorption and retention of $\mathrm{Na}$.

Alternatively, the interstitial space is formed from proteoglycans, which are under a swelling pressure, restrained by collagen fibres. These sulphate polyanions, which are electrically but not osmotically active, could be disturbed either during synthesis, or sulphation, or subsequently by radical and aldehydic attack. The speed with which kwashiorkor can supervene suggests that it is the existing framework that is damaged. There is a greatly increased rate of spreading of intradermally injected liquids in malnutrition (Jewish Physicians of the Warsaw Ghetto, 1979), suggesting a local interstitial abnormality.

It is interesting to note that the 'epidemics' of oedema in India during the last century (see McCance, 195I), which were clinically similar to famine oedema and kwashiorkor, were caused by contaminated cooking oil: presumably this resulted in a peroxidative stress. It has even been suggested that the oedema of beriberi, which was not observed with experimental thiamin deficiency (Eijkman, 1929), requires the added stress of mycotoxicosis to produce oedema (Tainsh, 1984). Thiamin deficiency impairs NADPH production and so, if there was a concomitant increased flux of radicals, GSH would become depleted. A mechanism such as this would explain why deficiency of a single nutrient, thiamin, gives such startlingly different clinical pictures under different circumstances.

Apart from thiamin, considerations of the other nutrients involved in free radical protection and their place in glutathione metabolism may explain both why there are some clinical similarities between deficiencies of these nutrients and the features of kwashiorkor, and why experimental removal of these nutrients results in the production of individual signs of kwashiorkor when the deficiency is extreme. For example, extreme and prolonged dietary protein deficiency may well result in cysteine deficiency and a resulting lack of production of glutathione, without necessarily an increased demand for glutathione; they are, however, vulnerable so that the few animals that receive a radical stress will develop oedema. The reason why the experimental manoeuvres produce inconsistent results then becomes clear.

Fatty liver is the hallmark of all forms of peroxidation reaction in the liver, the distribution of fat depending on the mode of production of the radical. The hepatic mitochondrial membrane will be particularly vulnerable if there is a reduction in Mn superoxide dismutase and in GPX (Se) because these two enzymes provide the major protection from the intense oxygen metabolism in the mitochondria. 
Nausea, diarrhoea, vomiting and immunoincompetence, all characteristic, albeit non-specific, signs commonly found in kwashiorkor, are the cardinal symptoms of radiation injury, an unequivocal example of generalized free radical formation.

We conclude that, although there are individually other plausible aetiologies for the clinical features of kwashiorkor, each can be caused by an excess free radical stress in the face of inadequate protection, and that none of the other candidate hypotheses adequately account for the association between the different clinical features.

\section{Death from malnutrition}

Examination of the results we have described shows that those children who die have the lowest levels of vitamin E, GPX, $\mathrm{Zn}$, and glutathione; they have the highest levels of plasma ferritin and hepatic $\mathrm{Fe}$ (Waterlow, 1948). It was only in these desperately ill children who died that the proportion of GSSG to total glutathione was raised (up to $20 \%$ ); at the same time, these children had extraordinarily high activities of their hexose-monophosphate shunt enzymes (up to five times normal). In other words, the features which distinguish the children with kwashiorkor are all exaggerated in those who die from kwashiorkor.

Fig. 9 shows the admission erythrocyte GPX activity plotted against the admission plasma ferritin concentrations from a series of malnourished children. All the children who, on admission, had a GPX activity of below $17 \mathrm{U} / \mathrm{g}$ haemoglobin and a ferritin of above $25^{\circ} \mu \mathrm{g} / \mathrm{l}$, subsequently died: all except one child who did not fulfil these criteria survived. Thus we were able to accurately

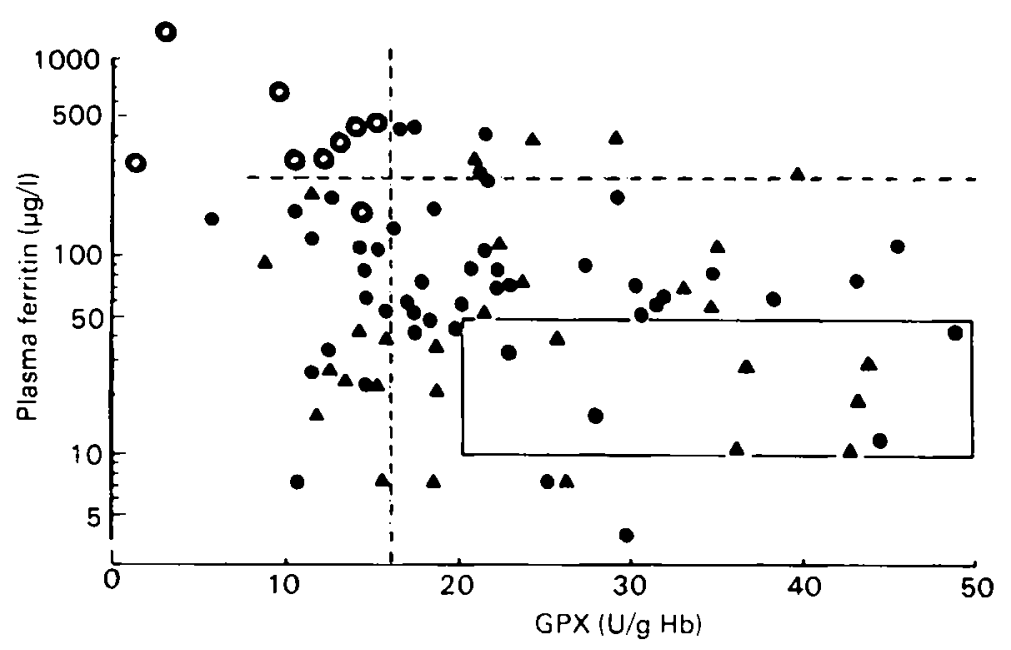

Fig. 9. Admission erythrocyte glutathione peroxidase ( $E C$ r.11.1.9: GPX) v. plasma ferritin in malnourished children. Each point represents a separate child. ( $\Delta$ ), marasmus; $(O)$. kwashiorkor: $(O)$. children who subsequently died. The block is the normal range. (- - -), arbitrarily drawn at GPX $17 \mathrm{U} / \mathrm{g}$ haemoglobin $(\mathrm{Hb})$ and ferritin $250 \mu \mathrm{g} / \mathrm{l}$. 
predict which children would subsequently die and which would survive on the basis of measuring two of the integral components of the free radical scheme. This is particularly impressive as most of the children did not succumb for a considerable time after the measurements were made. This opens the way for rational treatment to be instituted before the children become moribund.

Clearly many of the therapeutic manoeuvres that have been tried are inappropriate or even dangerous if the free radical hypothesis is correct. For instance, on the basis of the assumption that the children have an energy deficit as part of their syndrome, we have in the past advocated adding oil to the children's diet: it would be hazardous to give a child without free radical protection a diet high in polyunsaturated fatty acids. It is probably relevant that McFarlane et al. ( 1970 ) found that giving $\mathrm{Fe}$ to children with kwashiorkor resulted in a very high mortality rate. Giving protein hydrolysates intravenously also results in an extraordinarily high mortality rate (Gillman \& Gillman, 195 I).

On the other hand, if the present hypothesis is correct, there are a number of new therapeutic lines that could be very effective in treating and preventing kwashiorkor; they are all open to experimental testing and if they are found to be effective will provide very strong evidence in favour of the hypothesis.

We conclude that trials of $\mathrm{Fe}$ chelation, antioxidant therapy with both waterand fat-soluble agents, possibly allopurinol, as well as $\mathrm{Se}, \mathrm{Mn}, \mathrm{Zn}, \mathrm{Cu}$ and vitamin replacement therapy, with the avoidance of polyunsaturated fats, suppression of small bowel flora and active treatment of infections, in children with kwashiorkor are warranted.

This work would not have been possible without the continued support of the Wellcome Trust. Professor J. C. Waterlow, Dr. A. A. Jackson and Dr. B. E. Golden provided the atmosphere in which these ideas were germinated. We thank Mrs. L. Charley and Miss J. Foreman for their technical assistance and the nursing staff for their loving care of our unfortunate patients.

\section{REFERENCES}

Adams, E. B. \& Scragg. J. N. (1965). British Fournal of Haematology I I, 676-68I.

Bennett, F., Golden, M. H. N., Ramdath, D. \& Golden, B. E. $(1984)$.. West Indian Medical fournal 33 (Suppl.), 46.

Bors, W., Saran, M. \& Michel, C. (1982). International fournal of Radiation Biology 41, 403-501.

Burk, R. F., Pearson, W. N., Wood, R. P. II. \& Viteri, F. (1967). American fournal of Clinical Nutrition 20, 723-733.

Charley, L., Ramdath, D., Golden, M. H. N. \& Foreman, J. (1986). West Indian Medical fournal 35 (Suppl.), $5^{\circ}$.

Cheek, D. B., Hill, D. E., Cordano, A. \& Graham, C. G. (1970). Pediatric Research 4, I35-1 44.

Cooperstock, M. S., Tucker, R. P. \& Baublis, J. V. (1975). Lancet i, 1272-1274.

Coward, D. G. \& Whitehead, R. G. (1972). British Journal of Nutrition 28, 223-237.

Drasar, B. S. \& Hill, M. J. (1974). Human Intestinal Flora. New York: Academic Press.

Eijkman, C. (1929). Antinuritic Vitamin and Beriberi. Nobel Prize Lecture, i ith December 1929.

Esterbauer, H. (1982). In Free Radicals, Lipid Peroxidation and Cancer, pp. 101-128 [D. C. H. McBrian and T. F. Slater, editors]. New York: Academic Press. 
Fondu, P., Hariga-Muller, C., Mozes, N., Neve, J., Van Steirteghem, A. \& Mandekbaum, I. M. (1978). American Journal of Clinical Nutrition 31, 46-56.

Gabig, T. G. \& Babior, B. M. (1982). In Superoxide Dismutase, vol. 2, pp. I-15 [L. W. Oberly, editor]. Boca Raton: C.R.C. Press.

Gillman, J. \& Gillman, T. (1951). Perspectives in Human Nutrition. New York: Grune \& Stratton.

Golden, M. H. N. (1985). In Nutritional Adaptation in Man, pp. 169-187 [K. Blaxter and J. C. Waterlow, editors]. London: Applied Science Publishers.

Golden, M. H. N., Golden, B. E. \& Bennett, F. I. (1985). In Trace Element Metabolism in Man and Animals, vol. 5, pp. 775-779 [C. F. Mills, I. Bremner and J. K. Chesters, editors]. Slough: Commonwealth Agricultural Bureaux.

Gopalan, G. (1968). In Calorie Deficiencies and Protein Deficiency, pp. 48-58 [R. A. McCance and E. M. Widdowson, editors]. London: Churchill.

Halliwell, B. \& Gutteridge. J. M. C. (1984). Biochemical Gournal $219,1-4$.

Hassan, H., Hashim, S. A., Van Itallie, T. B. \& Sebrell, W. H. (I966). American fournal of Clinical Nutrition 19, $147^{-1} 57$.

Hendrickse, R. G. (1984). Transactions of the Royal Society of Tropical Medicine and Hygiene 78, 427-435.

Heyworth, B. \& Brown, J. (1975). Archives of Disease in Childhood 50, 27-33.

Hibbert, J. M. \& Golden, M. H. N. (1981). Fournal of Tropical Paediatrics 27, $255^{-258 .}$

Jewish Physicians of the Warsaw Ghetto (1979). Hunger Disease. [M. Winick, editor]. New York: John Wiley.

Kaplay, S. S. (1978). American fournal of Clinical Nutrition 31, 574-584.

Levine, R. J. \& Olson, R. E. (1970). Proceedings of the Society of Experimental Biology and Medicine 134, 1030-1034.

McCance, R. A. (I95I). Studies of Undernutrition, Wuppertal 1946-9. Medical Research Council Special Report Series no. 275. London: H. M. Stationery Office.

McCord, J. M. \& Petrone, W. F. (1982). In Lipid Peroxides in Biology and Medicine, pp. 123-1 31 [K. Kagi, editor]. New York: Academic Press.

McFarlane, H., Reddy, S., Adcock, K. J., Adeshina, H., Cooke, A. R. \& Akene. J. (1970). British Medical Yournal 3, 268-720.

McLaren, D. S., Faris, R. \& Zekian, B. (1968). Fournal of Tropical Medicine and Hygiene $7 \mathrm{r}$, $271-281$.

McLaren, D. S., Shirajian, E., Loshkajian, H. \& Shadarevian, S. (1969). American fournal of Clinical Nutrition 22, $863-870$.

Mead, J. F., Wu, G.-S. \& McElhaney, R. N. (1982). In Lipid Peroxides in Biology and Medicine, pp. I6I-1 78 [K. Kagi, editor]. New York: Academic Press.

Morehead, C. O., Morehead, M., Allen, D. M. \& Olson, R. E. (1974). Fournal of Tropical Paediatrics 20, 14 I-147.

Morley, D. (1964). Proceedings of the Royal Society of Medicine 57, 846-849.

Mukherjee, K. L. \& Sarkar, N. K. (1958). British fournal of Nutrition 12, 1-7.

Nichols, B. L., Alleyne, G. A. O., Barnes, D. J. \& Hazelwood, C. F. (1969). Gournal of Pediatrics 74, $49-57$.

Patrick, J. \& Golden, M. H. N. (1977). American fournal of Clinical Nutrition 30, 1478-148r.

Pereira, S. M. \& Begum. A. (1971). Archives of Disease in Childhood 46, 525-527.

Rowland, M. G. M., Barrel, R. A. E. \& Whitehead, R. G. (1978). Lancet i, 136-138.

Slater, T. F. (1984). Biochemical fournal 222, I-15.

Srikantia, S. G. (1958). Lancet i, 667-668.

Tainsh, A. R. (1984). Nutrition and Health 3, 189-193.

Tietze, F. (1969). Analytical Biochemistry 27, 502-522.

Warren, P. J., Hansen. J. D. I. \& Lehmann, B. H. (1969). Proceedings of the Nutrition Society 28, $6 A-7 \Lambda$.

Waterlow, J. C. (1948). Fatty Liver Disease in Infants in the British West Indies. Medical Research Council Special Report Series no. 263. London: H.M. Stationery Office.

Wellcome Trust Working Party. (1970). Lancet ii, 302-303.

Whitehead, R. G. (1979). Zeitung Ernahrungswiss 23 (Suppl.), 72-84.

Williams, C. D. (1935). Lancet ii, $1151-1152$.

Printed in Great Britain 